\title{
Artery and Vein Classification in Retinal Images using Graph Based Approach
}

\author{
Ritesh R. Khatakalle ${ }^{1}$, Prof. R. U. Shekokar ${ }^{1}$ \\ Department of Electronics \& Telecommunication, R.M.D Sinhgad School of Engineering, Pune, India ${ }^{1}$
}

\begin{abstract}
Digital image analysis of eye fundus images has several benefits than current observer based techniques. The characteristic symptoms of different systemic diseases like hypertension, glaucoma, diabetes and cardiovascular disorder etc. Affects retinal vessels. Diseases like diabetes show abnormalities and diameter changes in retinal blood vessels. In hypertension retinal blood vessels show dilatation and elongation of main arteries and veins. Arteriolar to venular diameter ratio (AVR) express high blood pressure levels, diabetic retinopathy and retinopathy of prematurity. Among other image processing operations the estimation of AVR requires vessel segmentation, accurate vessel width measurement and artery or vein classification [1].Hence the identification of arteries is essential to detect eye diseases. The work has been done on automated classification of retinal vessels and hence it is a challenging task.
\end{abstract}

Keywords: Artery/vein classification, graph, retinal images, vessel segmentation.

\section{INTRODUCTION}

Nowadays for image analysis graph based methods have been used which are useful for retinal vessel segmentation, retinal image registration and retinal vessel classification [2]. The segmented vessels are analyzed using type of intersection and then assigned artery or vein labels to each vessel segment. So the combination of labels and intensity features decides final artery or vein class. Most of the methods uses intensity features to differentiate between arteries and veins. Due to the acquisition process, very often the retinal images are no uniformly illuminated and exhibit local luminosity and contrast variability, which can affect the performance of intensity-based $\mathrm{A} / \mathrm{V}$ classification methods. For this reason,proposed method which uses additional structural information extracted from a graph representation of the vascular network. The results of the proposed method will show improvements in overcoming the common variations in contrast inherent to retinal images.

\section{LITERATURE REVIEW}

With respect to said work an extensive literature survey is conducted accordingly which is presented as below,

1. Several features like visual and geometrical have explored the methods for artery or vein classification. Vessel diameter is not a reliable feature for artery or vein classification since it can be affected by diseases [3]. 2. Martinez- Perez et al. (2002) Insemi automatic method pixel.

[4] geometrical and topological features of single vessel 7. R.Estrada, C.Tomasi et al.(2012) present a segments and sub trees are calculated. Significant methodology[9] for vessel structure in human retina using pointsare detected through the skeleton extracted from the Dijkstra's shortest-path algorithm. The method requires no

segmentation result. For labeling purposeroot segment of manual intervention, preserves vessel thickness and the tree is tracked and then algorithm will search for its unique terminal points and decide if the segment is artery or vein.

3. Grisan et al.(2003) In optic disc zone arteries rarely cross arteries and veins rarely cross veins[5] hence by using vessel structure represented by tracking the classification is propagated outside this zone where little information is available to discriminate between arteries and veins. By applying 'a divide at impera' approach which partitioned a concentric zone around the optic disc into quadrants performs more robust local classification analysis.

4. S.Vazquez et al.(2009) In color based clustering algorithm with a vessel tracking method[6] retinal images are divided into four quadrants and then it combines the result. Then by using tracking strategy based on minimal path vessel segments are joined to support the

classification by voting.

5. C. Kondermann, D. Kondermann et al.(2007) Two feature extraction methods and two classification methods[7], based on support vector machine and neural network to classify retinal vessels. One of the feature extraction methods is based on ROI (Region Of Interest) around each centerline point while the other is profile based. To reduce dimensionality of feature vectors principal component analysis is used.

6. M. Niemeijer, B. van Ginneken et al.(2009) Image feature and classifier is an automatic method for classifying retinal vessels into arteries and veins[8]. A set of centreline features is extracted and a soft label is assigned to each centerline, indicating it's being a vein follows vessel branching naturally and efficiently.

8. M. Niemeijer, X. Xu, A. Dumitrescu, P. Gupta et al.(2011) In the classification method[10] is considered as 
a step in calculating AVR value. The estimation of AVR requires vessel segmentation, accurate vessel width measurement and artery vein classification hence slight error can produce large influence on the final value.

\section{PROPESED WORK}

The method proposed in this project follows a graph based approach, where the characteristics of the vessel tree at the region near the optic disc are focused. In this region arteries and veins rarely cross themselves which helps to define different types of intersection points: A bifurcation, crossing, meeting and connecting point. The geometrical analysis of the graph representation of the vascular structure takes the decision on the type of intersection point.

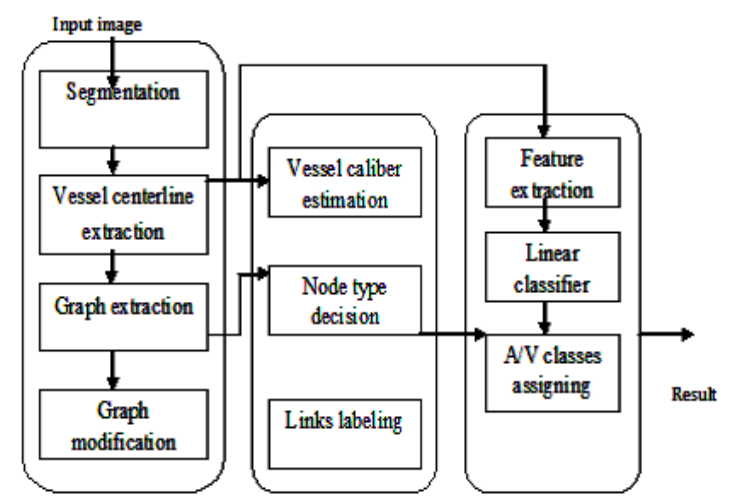

Fig. 1: Proposed block diagram of A/V Classification

Fig. 1 shows the block diagram of the proposed method for artery or vein classification. The method has the following phases. 1) Graph generation 2) Graph analysis 3) Vessel classification. The method first extracts a graph from the vessel structure and then makes a decision on the type of intersection point i.e. graph node. Based on the node type in each subgraph vessel segments are identified and then labelled using two distinct labels such as artery or vein. The details of the each phase are as follows.

\section{1) Graph generation:}

For generating the graph following three steps are used.

i) Vessel segmentation: This method follows a pixel processing-based approach such as pre-processing has, where the intensity is normalized by subtracting an estimation of the image background, obtained by filtering with a large arithmetic mean kernel. In the next phase, centreline candidates are detected using information provided from a set of four directional Difference of Offset Gaussian filters, then connected into segments by a region growing process, and finally these segments are validated based on their intensity and length characteristics. The third phase is vessel segmentation, where multiscale morphological vessel enhancement and reconstruction approaches are followed to generate binary maps of the vessels at four scales.

ii) Vessel centreline extraction: An iterative thinning algorithm is used to obtain centreline image which then applied to the vessel segmentation result. This phase removes border pixels until the object shrinks to a minimally connected stroke. This is shown in fig.2(c).
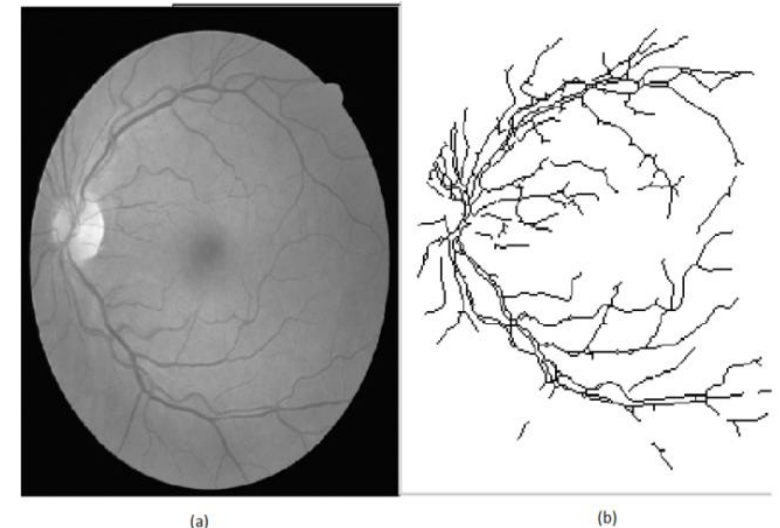

Fig. 2:Graph generation.(a)Original image; (b)Vessel segmentation

iii) Graph extraction: This step involves extraction of graph nodes from the centreline image by finding the intersection points and the endpoints. To find the links between nodes, all the intersection points with their neighbours are removed from the centreline image and image with separate vessel segments is recovered.fig2(d) is obtained from centreline image of fig2(c).

iv) Graph modification: Extracted vascular structure may contain following graphical errors.

- the splitting of one node into two nodes

- missing a link on one side of node

- False link.

If these errors are occurred the extracted graph should be modified.

2) Graph Analysis: In graph analysis phase the type of node is identified. But in this phase we are not confirming abouteach label corresponds to an artery or vein class. There are four different types of nodes. These are classified based on number of links connected to each node, the orientation of each link, angles between the links, the vessel calibre at each link and the degree of adjacent node. These are as follows.

1. Connecting point: This is the point where vessels never cross each other; however these are the continuation nodes connecting different segments of the same vessel.

2. Crossing point: At this point artery and veins cross each other.

3. Bifurcation point: A bifurcation point is the intersection point where a vessel bifurcates to a narrower part.

4. Meeting point: Two different types of are very close to each other and meet each other without crossing. Here the vessels exactly end on the other vessel. In the proposed 
system node analysis is divided into different cases. The nodes under analysis are represented by gray dots and other nodes are represented as black dots, except the endpoints which are represented as white dots. Solid lines show the links for one label and the dashed lines represent the other label.

Intersection Node: In this point the vessel will be crossed each other at this point we give name an intersection node. It represents with blue square box. It shown in fig.3(a).

Spliting Node: At this point the vessel split with main vessel line and ends with an end point. It represents with a green square box. It shown in fig. 3(b).

End Node: In this node format it will show the exact end point of the vessel. It represents with red square box. It shownin fig. 3(c)
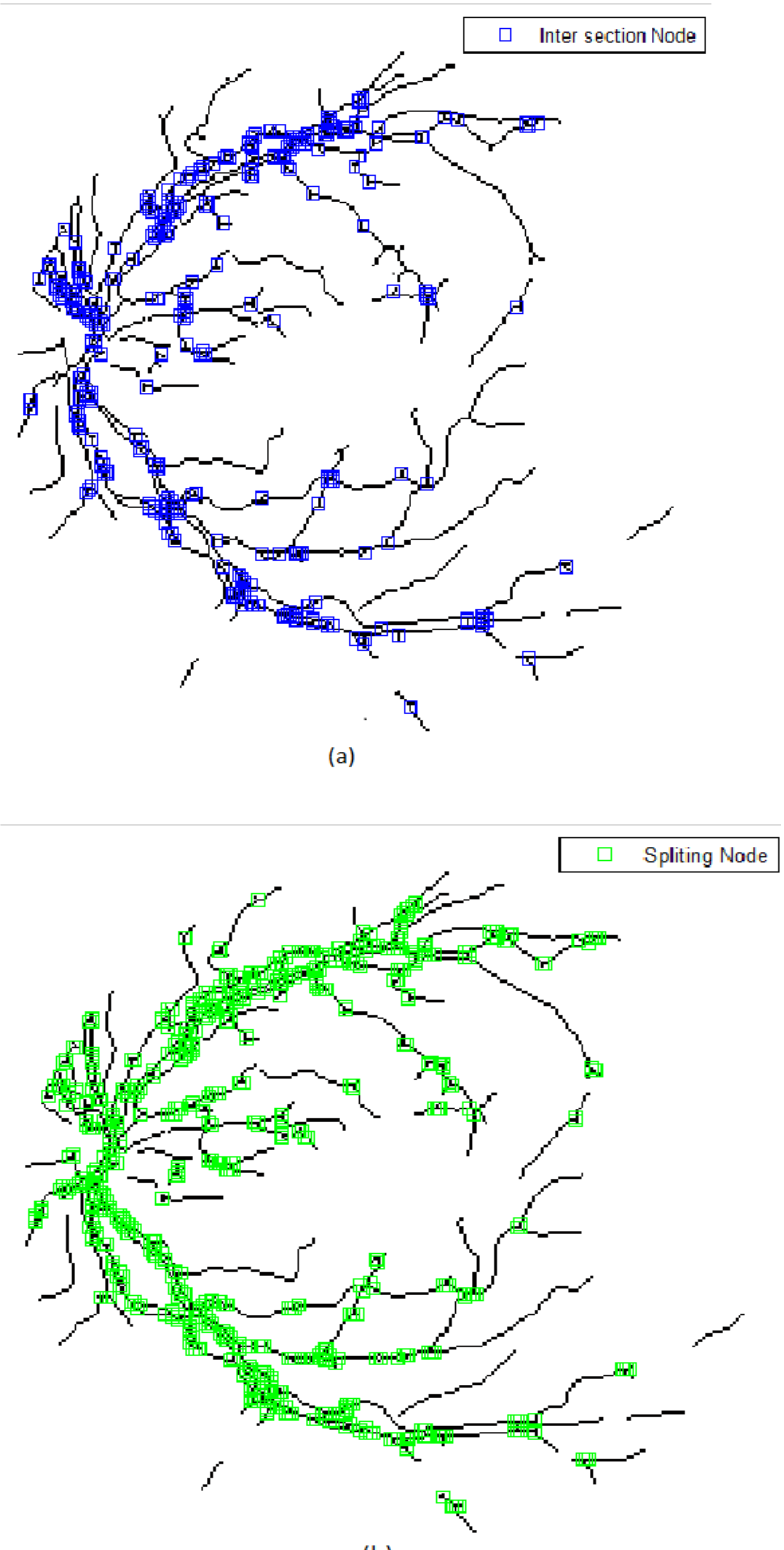

(b)

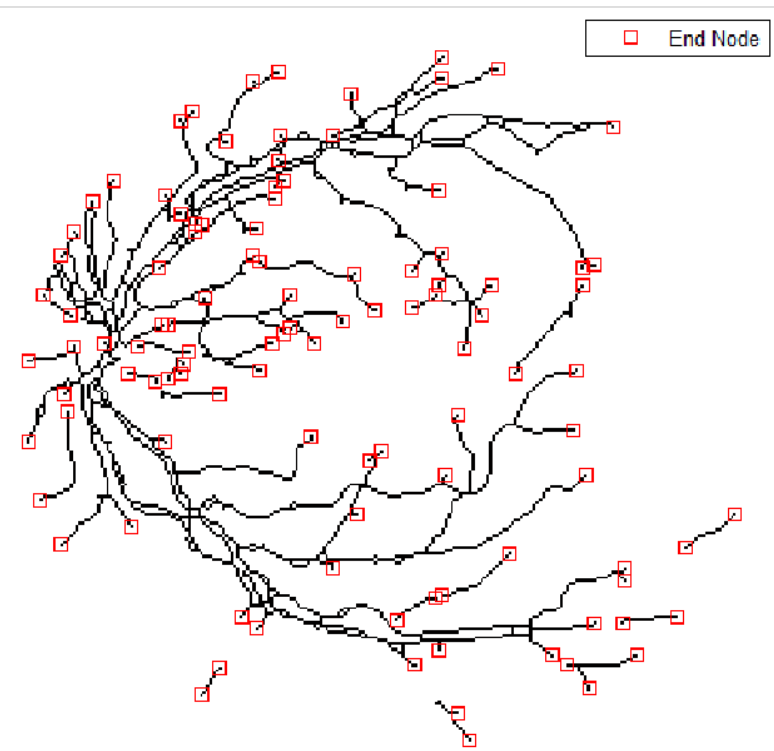

(c)

Fig. 3.Node Generation, (a) intersection node; (b)splitting node; (c) endnode

\section{IV.ARTERY/VEIN CLASSIFICATION}

Based on the above labeling phase information final task is to assign the artery class(A) to one of the labels, and the vein class $(\mathrm{V})$ to the other. This can be done by adding structural information to the vessel intensity information. Each centreline pixel is measured and normalized to zeromean and unit standard deviation with 30 features given below.

Letter followed by a period. For example, see heading " $\mathrm{C}$. Section Headings" above.

Level-3 Heading: A level-3 heading must be indented, in Italic and numbered with an Arabic numeral followed by a right parenthesis. The level-3 heading must end with a colon.

Table: List of features measured for each centerline Pixel

\begin{tabular}{|c|c|}
\hline Nr. & Features \\
\hline $1-3$ & $\begin{array}{l}\text { Red, Green and Blue intensities of the } \\
\text { centerline pixels. }\end{array}$ \\
\hline $4-6$ & $\begin{array}{l}\text { Hue, saturation and intensities of the } \\
\text { centerline pixels. }\end{array}$ \\
\hline $7-9$ & $\begin{array}{c}\text { Mean of Red, Green and Blue intensities in } \\
\text { the vessel. }\end{array}$ \\
\hline $10-12$ & $\begin{array}{l}\text { Mean of Hue, Saturation and Intensity in the } \\
\text { vessel }\end{array}$ \\
\hline $13-15$ & $\begin{array}{l}\text { Standard deviation of Red, Green and Blue } \\
\text { intensities in the vessel. }\end{array}$ \\
\hline 16-18 & $\begin{array}{l}\text { Standard deviation of Hue, Saturation and } \\
\text { Intensity in the vessel }\end{array}$ \\
\hline $19-22$ & $\begin{array}{l}\text { Maximum and minimum of Red and Green } \\
\text { intensities in the vessel }\end{array}$ \\
\hline $23-30$ & $\begin{array}{c}\text { Intensities of the centerline pixel in a } \\
\text { Gaussian blurred }(\sigma=2,4,8,16) \text { of Red and } \\
\text { Green Plane }\end{array}$ \\
\hline
\end{tabular}


In proposed system the features are tested using different linear classifiers. For feature selection, we use sequential forward floating selection, which starts with an empty feature set and adds or removes features when this improves the performance of the classifier. The trained classifier is used for assigning the $\mathrm{A} / \mathrm{V}$ classes to each one of the sub graph labels.

\section{CONCLUSION}

After completion of the work following objectives are accomplished,

1. Collection of the raw image from the database.

2. Extraction the vessel segments from rest of the image.

3. Extracting a graph from the vascular structure.

4. Take a decision on the type of each intersection point (Graph node).

5. Identification of vessel segments (Graph link) that belongs to a particular vessel and label using two distinct labels.

6. Extracting a set of features and using a linear classifier assign Artery or Vein class.

\section{REFERENCES}

[1] M. D. Knudtson, K. E. Lee, L. D. Hubbard, T. Y. Wong, R. Klein, and B. E. K. Klein,"Revised formulas for summarizing retinal vessel diameters," Current Eye Res., vol. 27, pp.143-149, Oct. 2003

[2] R. Estrada, C. Tomasi, M. T. Cabrera, D. K. Wallace, S. F. Freedman, and S. Farsiu, "Exploratory dijkstra forest based automatic vessel segmentation: Applications in videoindirect ophthalmoscopy (VIO)," Biomed. Opt. Exp., vol. 3, no. 2, pp. 327 339,2012

[3] K. Rothaus, X. Jiang, and P. Rhiem, "Separation of the retinal vascular graph in arteries an veins based upon structural knowledge," Image Vis.Comput., vol. 27, pp. 864-875, Jun.2009.

[4] M. E. Martinez-Perez, A. D. Hughes, A. V. Stanton, S. A.Thom, N. Chapman, A. A. Bharath, and K. H. Parker, "Retinal vascular tree morphology: A semi-automaticquantification," IEEE Trans. Biomed. Eng., vol. 49, no. 8, pp. 912-917, Aug. 2002.

[5] E. Grisan and A. Ruggeri, "A divide et impera strategy forautomatic classification of retinal vessels into arteries and veins," in Proc. 25th Annu. Int. Conf. IEEE Eng. Med. Biol. Soc., Sep. 2003, pp. 890-893.

[6] S. Vazquez, B. Cancela, N. Barreira, M. Penedo, and M.Saez, "On the automatic computation of the arterio-venousratio in retinal images: Using minimal paths for the artery/vein classification," in Proc. Int. Conf. Digital ImageComput., Tech. Appl., 2010, pp. 599604.

[7] C. Kondermann, D. Kondermann, and M. Yan, "Blood vessel classification into arteries and veins in retinal images," Proc. SPIE, Progr.Biomed. Opt. Imag., vol. 6512,no. 651247, Feb. 2007.

[8] M. Niemeijer, B. van Ginneken, and M. D. Abramoff,

[9] "Automatic classification of retinal vessels into arteries andveins," Proc. SPIE, Progr.Biomed. Opt. Imag., vol. 7260,no.72601F, Feb. 2009.

[10] R. Estrada, C. Tomasi, M. T. Cabrera, D. K. Wallace, S. F.Freedman, and S. Farsiu,"Exploratory dijkstra forest basedautomatic vessel segmentation: Applications in videoindirect ophthalmoscopy (VIO)," Biomed. Opt. Exp., vol. 3,no. 2, pp. 327339, 2012.

[11] M. Niemeijer, X. Xu, A. Dumitrescu, P. Gupta, M. A. B.van Ginneken, and J. Folk, "Automated measurement of thearteriolar-tovenular width ratio in digital color fundusphotographs," IEEE Trans. Med. Imag., vol. 30, no. 1, pp. 1941-1950, Nov. 2011

\section{BIOGRAPHIES}
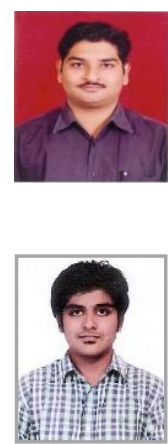
Coordinator in E \&Tc Department. Maharashtra, India.
Mr. Ritesh R. Khatakalle received BE degree from Shivaji University. He is now pursuing for ME (VLSI \& Embedded) from SavitribaiPhule Pune University, Pune

Prof. R. U. Shekokar is a Assistant professor in Department of Electronics and telecommunication, RMD Sinhgad school of Engineering, Savitribai Phule University, Pune. He is ME (Electronics), having 13 years of teaching experience. $\mathrm{He}$ is a $\mathrm{PG}$ 